\title{
Surface-Confined Metal-Organic Nanostructures from Co-Directed Assembly of Linear Terphenyl-dicarbonitrile Linkers on $\operatorname{Ag}(111)$
}

\author{
U. Schlickum, ${ }^{*},{ }^{\dagger}$ F. Klappenberger ${ }^{\ddagger}$ R. Decker, ${ }^{\dagger}$ G. Zoppellaro ${ }^{\S}$ S. Klyatskaya, ${ }^{\S}$ M. Ruben, ${ }^{*, \S}$ \\ K. Kern, ${ }^{\dagger, l}$ H. Brune, ${ }^{\dagger}$ and J. V. Barth ${ }^{*, *}$ \\ Institute of Condensed Matter Physics, Ecole Polytechnique Fédérale de Lausanne, CH-1015 Lausanne, Switzerland, \\ Physik Department E20, Technische Universität München, D-85748 Garching, Germany, Institut für \\ Nanotechnologie, Karlsruher Institut für Technologie, D-76021 Karlsruhe, Germany, and Max-Planck-Institut \\ für Festkörperforschung, D-70569 Stuttgart, Germany
}

Received: May 18, 2010; Revised Manuscript Received: August 12, 2010

\begin{abstract}
A detailed structural analysis of the surface supported self-assembly of terphenyl-4,4"-dicarbonitrile molecules $\left(\mathrm{NC}-\mathrm{Ph}_{3}-\mathrm{CN}\right)$ linked by $\mathrm{Co}$ adatoms on $\mathrm{Ag}(111)$ reveals different surface patterns depending on the constraints applied to the system. Without constraints, i.e., sufficient mobility and absence of space limitations at the surface, extended regular honeycomb nanomeshes are formed. On the basis of high-resolution scanning tunneling microscopy images, an atomistic model is derived showing the crystallographic orientation of the molecules and a commensurate alignment of the honeycomb networks, which exist in two rotational domains on the $\mathrm{Ag}(111)$ atomic lattice. For Co deficiency, an additional star-like Co-directed motif has been identified, and fully disordered networks are present if space limitations are imposed. In these cases, nodal motifs exist showing between 3- and 6-fold coordination of Co centers.
\end{abstract}

\section{Introduction}

In the past decade, the self-assembly of organic and metalorganic architectures on crystalline surfaces has attracted widespread interest. ${ }^{1-4}$ This versatile approach provides a multitude of supramolecular structures with prospects for novel functionalities, notably when metal centers are incorporated that can be controlled by a specific molecular ligand environment. ${ }^{5,6}$ In particular, the fabrication of nanoporous networks became an important research topic, because such structures present templates and offer well-defined spaces for host-guest chemistry. ${ }^{7-11}$ Thus, different bottom-up protocols have been developed to realize two-dimensional (2D) open systems presenting a controlled cavity size, using hydrogen-bonding, ${ }^{12-16}$ metal-directed assembly, ${ }^{17-26}$ and the organization of flexible species. ${ }^{27-30}$ Moreover, beyond the self-assembly approach, covalent chemical reactions have been explored. ${ }^{31,32}$

Recently, we showed that polyphenyl-4,4"-dicarbonitrile building blocks ( $\mathrm{NC}-\mathrm{Ph}_{n}-\mathrm{CN}$, with $\left.n=3-6\right)$ form wellordered organic patterns on the $\operatorname{Ag}(111)$ surface, ${ }^{33,34}$ which can be used as precursors to realize highly regular metallosupramolecular nanomeshes following subsequent exposure to cobalt atoms. $^{35,36}$ The nanoporous honeycomb structure features an unusual 3-fold coordination of $\mathrm{CN}$ moieties to cobalt centers in the ideal network with a metal-linker stoichiometry of 2:3. ${ }^{35-37}$ As shown for the para-hexaphenyl-dicarbonitrile linkers, ${ }^{36}$ an epitaxial fit between honeycomb and atomic $\operatorname{Ag}(111)$ lattice promotes layers with excellent regularity.

Herein, we present a systematic investigation of the cobaltdirected assembly of the terphenyl-4,4"-dicarbonitrile linker $\left(\mathrm{NC}-\mathrm{Ph}_{3}-\mathrm{CN}\right)$ with varying stoichiometries and surface densi-

\footnotetext{
* To whom correspondence should be addressed. E-mail: u.schlickum@ fkf.mpg.de. Phone: +49 (0) 7116891539 . Fax: +49 (0) 7116891662.

† Ecole Polytechnique Fédérale de Lausanne.

* Technische Universität München.

$\S$ Karlsruher Institut für Technologie.

" Max-Planck-Institut für Festkörperforschung.
}

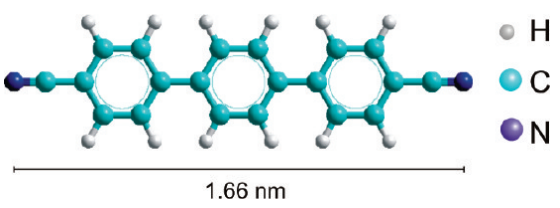

Figure 1. Schematic drawing of the terphenyl-4,4"-dicarbonitrile molecule. The indicated length is calculated for the planar configuration in the gas phase using HYPERCHEM calculations.

ties using low-temperature scanning tunneling microscopy (STM). By high-resolution STM measurements, we determine the orientation between the molecular linkers and the atomic $\operatorname{Ag}(111)$ lattice and propose a model showing the epitaxial fit of the superlattice on the surface, which is supported by complementary low-energy electron diffraction data. Under conditions of Co deficiency, we identify star-like Co-linker motifs with a 6-fold coordination of $\mathrm{CN}$ groups to a single $\mathrm{Co}$ center. Furthermore, by imposing space limitations in the metal-organic network formation, we realized fully reticulated disordered networks with coordination numbers varying between 3 and 6. Local deviations from the preferred 3 -fold coordination with characteristic defects were also found when kinetic limitations interfered in the nanomesh formation.

\section{Results and Discussion}

The employed molecular linkers, consisting of a linear chain of three phenyl rings with functional carbonitrile groups at both ends (Figure 1), are deposited by organic molecular beam epitaxy onto atomically clean and flat $\mathrm{Ag}(111)$ surfaces kept at $300 \mathrm{~K}$. Co atoms were provided from an e-beam source. After the deposition, the samples were cooled down to about $10 \mathrm{~K}$ for imaging the surface structures with a home-built low temperature scanning tunneling microscope (STM). ${ }^{38}$

An overview STM image is presented in Figure 2a showing four Ag terraces. Standing wave patterns are present on three 

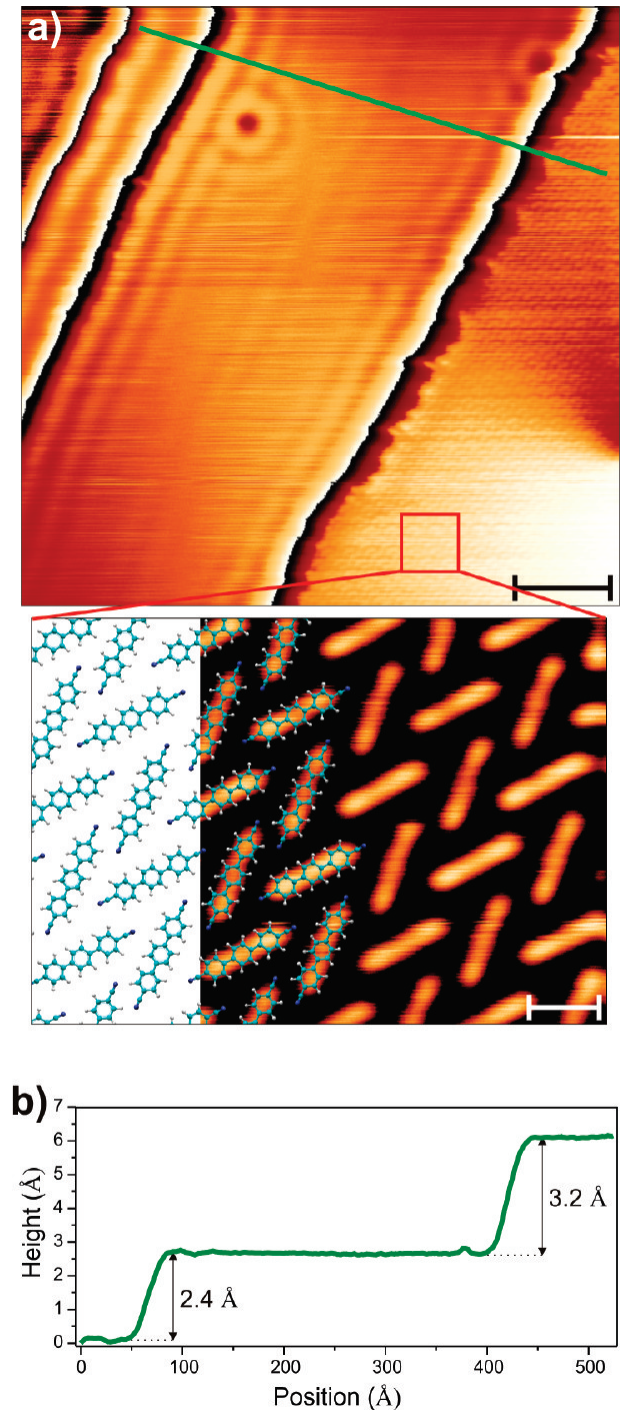

Figure 2. (a) Overview STM image (length of the scale bar: $10 \mathrm{~nm}$ ), showing the global arrangement of the molecules on the $\operatorname{Ag}(111)$ surface (tunnel current: $0.1 \mathrm{nA}$, bias voltage: $0.05 \mathrm{~V}$ ). The inset shows a zoom into the molecular domain demonstrating the close packed chevron pattern with a molecular model superimposed (length of the scale bar: $1 \mathrm{~nm}$; tunnel current: $0.1 \mathrm{nA}$, bias voltage: $-0.1 \mathrm{~V}$ ). (b) Line scan taken along the green line in part a, presenting the difference in step height of molecule free terraces and terraces covered by molecular domains.

terraces formed by the scattering of the surface state electrons at the step edges and two impurities. ${ }^{39}$ However, the standing wave pattern is absent on the right terrace. The line profile shows a monatomic $\operatorname{Ag}(111)$ step between terraces showing standing waves, while the apparent height between the middle and right terrace is about $0.8 \AA$ higher. A closer look at the structure on the fourth terrace (see inset in Figure 1a) shows the densely packed chevron pattern formed by the $\mathrm{NC}-\mathrm{Ph}_{3}-\mathrm{CN}$ molecules. ${ }^{33}$ Because several $\mathrm{Ag}$ terraces are completely free of molecules and others are completely covered, the STM image demonstrates that at $300 \mathrm{~K}$ the molecules are very mobile and that they prefer to form large single domains. During the cooldown process, the molecules freeze in their energetic most favorable position, the chevron pattern. The large single domains are an indication that it is energetically preferred to form $\mathrm{N} \cdots \mathrm{H}$ bonds and to avoid nonbonded carbonitrile groups. As discussed in ref 33 , the molecular structure of the chevron pattern is determined by the $\mathrm{N} \cdots \mathrm{H}$ bonds between adjacent ligands and the epitaxial fit of the organic molecules to the underlying $\operatorname{Ag}(111)$ surface lattice.

The subsequent deposition of Co atoms onto the organic phase on $\mathrm{Ag}(111) \mathrm{kept}$ at $300 \mathrm{~K}$ leads to the formation of long-range ordered honeycomb nanomeshes (demonstrated in ref 35). As visible in the overview STM topograph in Figure 3a, the honeycomb nanomeshes grow in domains that can reach $\mu \mathrm{m}^{2}$ large areas with a low defect concentration. The bright spots are impurities that are exclusively located on top of the nanomesh; they do not affect the underlying network structure. As indicated by purple lines, the boundaries of the nanomesh domains are straight and only angles of $30^{\circ}$ appear between them, reflecting the 6-fold symmetry of the honeycombs and the underlying $\operatorname{Ag}(111)$ surface lattice. Figure $3 b$ shows a highresolution STM image with molecular and within one hexagonal cavity atomic resolution of the $\mathrm{Ag}(111)$ lattice. We assume that the atomic contrast is produced by an adsorbed species that is trapped in the cavity but confined under the tip apex whenever the tip is scanned over this region. While in most STM images the Co atoms in the coordination nodes are not visible, they appear here as weak bright spots in the middle of the nodes. The imaging of the atomic substrate lattice together with the molecular network pattern allows one to clearly identify the orientation of the organic ligands with respect to the Ag lattice. An angle of $30^{\circ}$ is observed between the long molecular axis and the close-packed atomic rows of the substrate oriented along the crystallographic $\langle 1-10\rangle$ directions. Thus, the molecules are aligned along the crystallographic $\langle 11-2\rangle$ directions. We observed two domains of the honeycomb lattice rotated by $30^{\circ}$ with respect to each other and separated by sharp domain walls (Figure 3c). Within a given domain the molecules are exclusively aligned along $\langle 1-10\rangle$ or $\langle 11-2\rangle$, similar to the orientations observed for the nanomesh formed by the longest dicarbonitrile molecules. ${ }^{36}$ The presence of the two rotational domains is supported by low-energy electron diffraction (LEED) data (see the Supporting Information).

Figure $3 \mathrm{~d}$ and e shows an atomistic model for the two domains of the honeycomb nanomesh. The size of the honeycombs was determined from STM images (see ref 35) and the crystallographic orientations from STM images and complementary LEED patterns. The absence of any moiré pattern and the presence of highly symmetric honeycombs supports a commensurate arrangement of the nanomesh on the $\operatorname{Ag}(111)$ lattice, as similarly demonstrated for para-hexaphenyl-dicarbonitrile-based networks. ${ }^{36}$ For the orientational domain where molecules follow the $\langle 11-2\rangle$ directions, the unit cell is determined to be $(12 \times 12)$ substrate unit lattice vectors. Notice that all $\mathrm{Co}$ atoms are adsorbed on identical lattice sites. As for the para-hexaphenyl-dicarbonitrile-based honeycomb nanomesh ${ }^{36}$ the adsorption position is observed on the hollow sites; we assume this is the case also for this system. For geometry reasons, the $30^{\circ}$ rotated domain has to be expanded by about $1 \%$ to be commensurate. In matrix notation, this domain has a $\left(\begin{array}{ll}7 & 7 \\ -7 & 14\end{array}\right)$ unit cell. Assuming that the linker length does not substantially change upon adsorption, ${ }^{13}$ we estimate a corresponding $\mathrm{Co}-\mathrm{N}$ bond length of about $1.7-1.8 \AA$ for the two phases.

If we dose under-stoichiometric quantities of Co to the organic phase, we observe the network structures presented in Figure 4. In the overview STM topograph (Figure 4a), we see in the middle the honeycomb nanomesh as a channel in the pure organic phase. From this image, we derive an indication of the growth mechanism of the honeycombs and chevron patterns. 

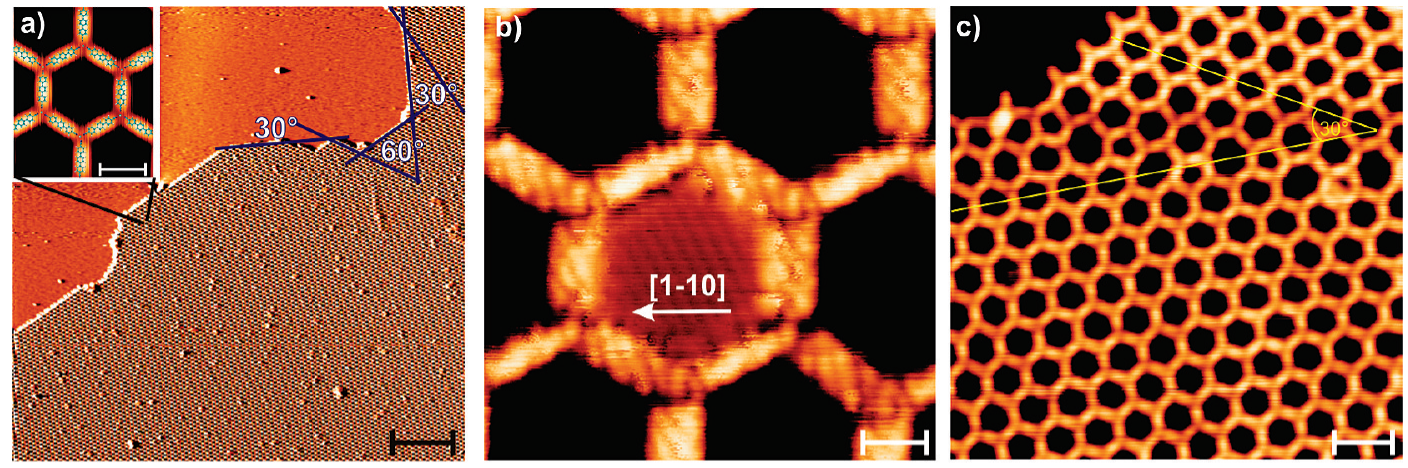

d)

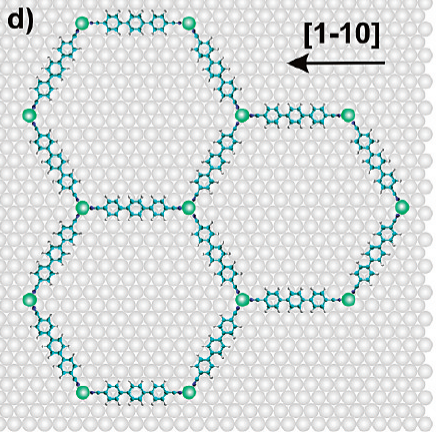

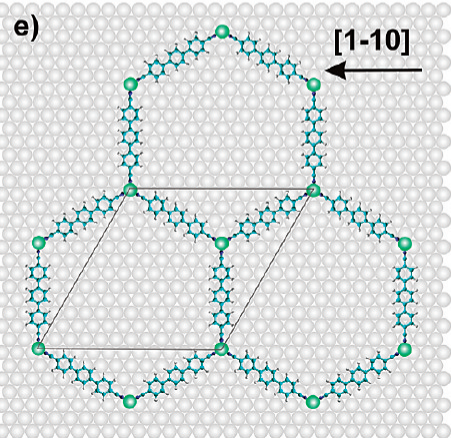

Figure 3. (a) Large scale STM image showing the long-range order of the honeycomb nanomesh (length of the scale bar: $50 \mathrm{~nm}$ ) and the directions of the domain boundaries (purple lines). The inset shows one hexagon with a molecular model superimposed (length of the scale bar: $2 \mathrm{~nm}$ ). (b) High-resolution STM image with molecular and atomic substrate resolution (length of the scale bar: $1 \mathrm{~nm}$ ). (c) Two honeycomb domains with a domain boundary (length of the scale bar is $5 \mathrm{~nm}$ ). ( $\mathrm{d}$ and e) Present atomistic models of the two orientational domains of the honeycomb nanomesh on the $\mathrm{Ag}(111)$ lattice. The inner diameter of the hexagons in parts d and e is 3.50 and $3.47 \mathrm{~nm}$, respectively. In part e, the unit cell is indicated in gray. Each gray sphere represents one Ag atom. The molecules are presented in the same color code as in Figure 1, and Co atoms are shown in green.

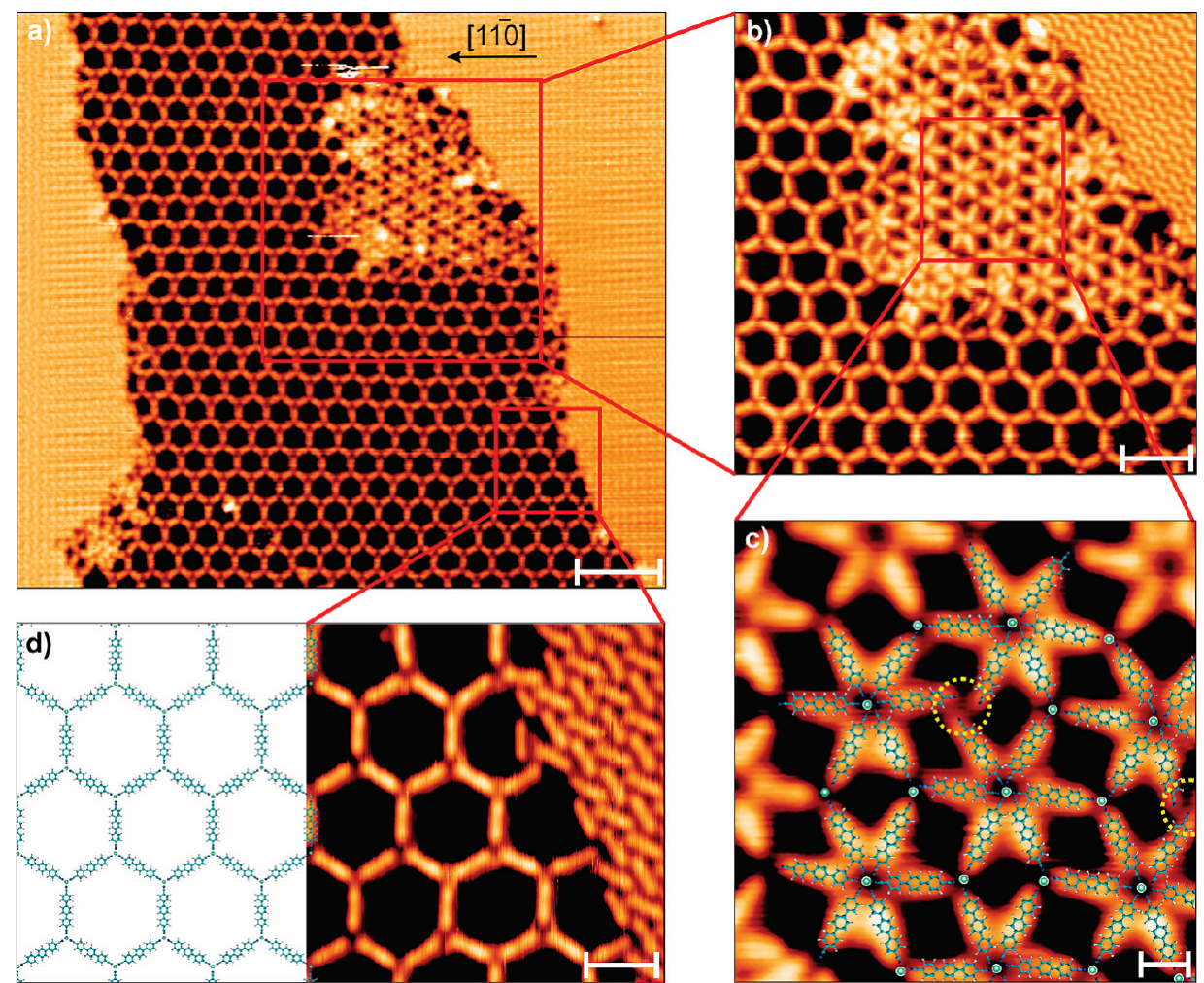

Figure 4. (a) Large scale STM image showing the surface architecture for an under-stoichiometric amount of Co atoms subsequently deposited onto the NC-Ph $3-\mathrm{CN} / \mathrm{Ag}(111)$ surface (length of the scale bar: $10 \mathrm{~nm}$ ). (b-d) Zoom into the different organic and metal-organic phases (length of the scale bars: (b) $5 \mathrm{~nm}$; (c) $1 \mathrm{~nm}$; (d) $2.5 \mathrm{~nm}$ ). Planar molecular models are superimposed.

We know that the organic molecules are very mobile at $300 \mathrm{~K}$, whereas the honeycomb nanomesh is stable at this temperature. When Co atoms are added to the organic phase at room temperature, they are coordinating the ligands into the 3-fold coordination motif, resulting in the formation of honeycomb nanomeshes. During the cooldown process, the molecules not 

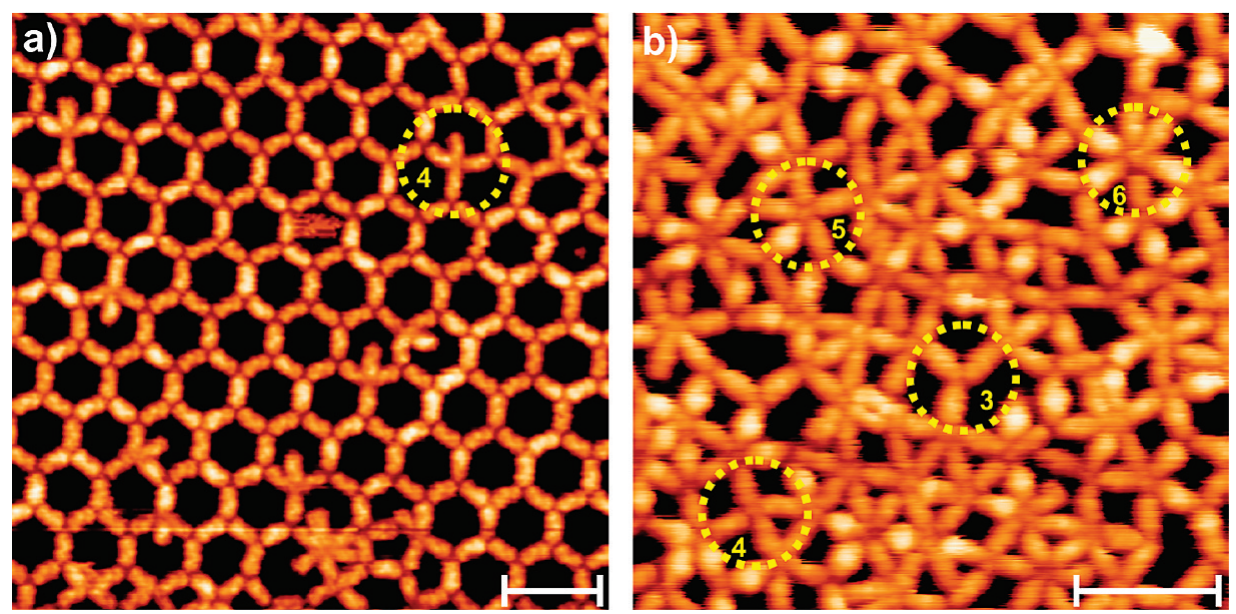

Figure 5. (a) Metal-organic network structure formed at $250 \mathrm{~K}$ (length of the scale bar: $5 \mathrm{~nm}$ ). Because of kinetic limitations, defects in the honeycomb order occur, frequently with local 4-fold coordination as indicated. (b) A disordered metal-organic layer evolves when space limitations obstruct the formation of the honeycomb nanomesh (length of the scale bar: $5 \mathrm{~nm}$ ). In this case, the sample was prepared at $300 \mathrm{~K}$. Several nodal motifs with 3- to 6-fold lateral $\mathrm{Co}$-carbonitrile coordination are marked.

being linked by Co atoms assemble around the honeycomb domains to minimize the number of nonbonded carbonitrile groups.

Very rarely, however, a third phase is present at surfaces with understoichiometric amounts of Co in which the molecules are arranged in a six-star configuration (Figure $4 \mathrm{~b}$ and c). This phase is only present at the boundary between the organic and metal-organic patterns. As in the honeycomb structure (Figure $4 \mathrm{~d}$ ), the distances between the $\mathrm{N}$ atoms of the molecules and the center point of the 6-fold coordination node reveal that also in the star configuration only one $\mathrm{Co}$ atom can be located in the node center. Therefore, in this special structure, one Co atom links six molecules together, as indicated in Figure 4c. The star configuration appears only for unsaturated samples, i.e., on surfaces without a sufficient amount of Co atoms to express 3 -fold linkages with all molecules. The molecules within this structure try to align along the high-symmetry $\langle 1-10\rangle$ directions. In the six-star configuration, typically one end of the molecules is connected to 6-fold coordinated Co centers, whereas the other one is 3 -fold coordinated. The star configuration is, however, not a completely symmetric phase like the chevron pattern and the honeycomb network. The angles between adjacent molecules deviate slightly from $60^{\circ}$. A closer look reveals that in some 3 -fold coordinated nodes molecules are aligned straight toward the node center, whereas in others they form a chiral node motif. Due to the dipole character of the dicarbonitrile group, ${ }^{33}$ the Co atom has to be present in the symmetric node motif-as for the honeycomb nanomesh-and absent in the chiral one-as for the pure organic phase. This irregularity of the nodal structure hinders the molecules from forming high symmetry six-star configurations. The nodes with missing Co atoms are visible in the STM data and highlighted by yellow circles in Figure 4c.

The described 6-fold lateral coordination represents the highest number identified to date for surface-supported coordination systems. It is ascribed to the surface bonding of the linkers promoting a flat adsorption geometry with the organic backbone parallel to the substrate. For comparison, only up to 4 -fold coordination was encountered in 2D iron or cobalt carboxylate compounds. ${ }^{40,41}$ This is presumably related to the steric limitations encountered with the usage of ditopic carboxylate moieties, that bind simultaneously to transition metal centers and surface atoms. ${ }^{42}$ Similar effects exist with pyridyl linkers, where the bulky functional group favors even a 2 -fold coordination at surfaces. ${ }^{43,44}$ With the present system, the energetic preference for 3 -fold coordination ${ }^{35}$ can be obviously overridden by the employed assembly conditions, whereby a necessary precondition is the "slenderness" of the functional carbonitrile end group.

The deposition of the organic ligands and the Co atoms at slightly lower temperatures $(250 \mathrm{~K})$ leads still to the formation of honeycomb nanomeshes, however, with a higher defect concentration (Figure 5a). At $250 \mathrm{~K}$, the molecular ligands are less mobile, and metastable coordination configurations can be found. They might be related to molecules which are caught in the hexagonal cavities during the formation of the honeycomb nanomesh. The result is the expression of an additional bond to the Co atoms sitting in the coordination nodes, resulting in local nodes with 4-fold coordination or other structural defects, similar to the stars described above.

In the case where more molecules are deposited onto the $\mathrm{Ag}$ surface than can be accommodated in a saturated honeycomb nanomesh monolayer, the molecules arrange in an irregular metal-organic pattern after the coevaporation of Co atoms at ambient temperature (cf. Figure 5b). In the STM topograph, motifs going from 3-fold up to 6-fold coordination can be found, as indicated by the yellow circles in the image. This provides a route toward a 2D random, fully reticulated coordination network which contrasts the usage of nonlinear dicarbonitrile linkers without packing constraints, reported recently. ${ }^{45}$

\section{Conclusion}

We present a detailed picture of the surface architectures and self-assembly processes present for $\mathrm{NC}-\mathrm{Ph}_{3}-\mathrm{CN}$ molecules coordinated by single Co adatoms on the $\operatorname{Ag}(111)$ surface. Appreciable surface mobility and absence of spatial constraints leads to the formation of highly regular metal-organic honeycomb nanomeshes. The high symmetry reflects a commensurability of the two rotational domains to the underlying $\operatorname{Ag}(111)$ lattice, and we present structural atomistic models for both of them. When additional constraints are imposed, such as Co deficiency, kinetic or spatial limitations, nodal motifs occur which exhibit from 3- up to 6-fold lateral $\mathrm{Co}$-carbonitrile coordination. However, only the 3 -fold coordination results in the formation of a highly symmetric surface architecture in extended mesoscale domains. 
Acknowledgment. We thank Willi Auwärter, Markus Etzkorn, Stefano Rusponi, Knud Seufert, Pardeep Thakur, and the European Synchrotron Radiation Facility for their help with the LEED and the room temperature STM image. Work was supported by the European Science Foundation Collaborative Research Programme FunSMARTs.

Supporting Information Available: LEED images and additional STM data. This material is available free of charge via the Internet at http://pubs.acs.org.

\section{References and Notes}

(1) De Feyter, S.; De Schryver, F. C. Chem. Soc. Rev. 2003, 32, 139

(2) Barth, J. V.; Weckesser, J.; Lin, N.; Dmitriev, S.; Kern, K. Appl. Phys. A 2003, 76, 645.

(3) Barth, J. V.; Costantini, G.; Kern, K. Nature 2005, 437, 671.

(4) Barth, J. V. Annu. Rev. Phys. Chem. 2007, 58, 375.

(5) Gambardella, P.; Stepanow, S.; Dmitriev, A.; Honolka, J.; Groot, F. d.; Gupta, S. S.; Sarma, D. D.; Bencok, P.; Stanescu, S.; Clair, S.; Pons, S.; Lingenfelder, M.; Lin, N.; Seitsonen, A. P.; Brune, H.; Barth, J. V.; Kern, K. Nat. Mater. 2009, 8, 189.

(6) Barth, J. V. Surf. Sci. 2009, 603, 1533. 203

(7) Cicoira, F.; Santato, C.; Rosei, F. Top. Curr. Chem. 2008, 285,

(8) Kudernac, T.; Lei, S.; Elemans, J. A. A. W.; De Feyter, S. Chem. Soc. Rev. 2009, 38, 402.

(9) Lin, N.; Stepanow, S.; Ruben, M.; Barth, J. V. Top. Curr. Chem. 2009, 287, 1 .

(10) Bonifazi, D.; Mohnani, S.; Llanes-Palles, A. Chem.-Eur. J. 2009 $15,7004-7025$

(11) Liang, H.; He, Y.; Ye, Y.; Xu, X.; Cheng, F.; Sun, W.; Shao, X.; Wang, Y.; Li, J.; Wu, K. Coord. Chem. Rev. 2009, 253, 2959.

(12) Griessl, S.; Lackinger, M.; Edelwirth, M.; Hietschold, M.; Heckl, W. M. Single Mol. 2002, 3, 25.

(13) Dmitriev, A.; Lin, N.; Weckesser, J.; Barth, J. V.; Kern, K. J. Phys. Chem. B 2002, 106, 6907 .

(14) Theobald, J. A.; Oxtoby, N. S.; Phillips, M. A.; Champness, N. R.; Beton, P. H. Nature 2003, 424, 1029.

(15) Stöhr, M.; Wahl, M.; Galka, C. H.; Riehm, T.; Jung, T. A.; Gade, L. H. Angew. Chem., Int. Ed. 2005, 44, 7394.

(16) Ruben, M.; Payer, D.; Comisso, A.; Gattinoni, C.; Lin, N.; Collin, J.-P.; Sauvage, J.-P.; De Vita, A.; Kern, K. J. Am. Chem. Soc. 2006, 128, 15644.

(17) Dmitriev, A.; Spillmann, H.; Lin, N.; Barth, J. V.; Kern, K. Angew. Chem., Int. Ed. 2003, 41, 2670.

(18) Spillmann, H.; Dmitriev, A.; Lin, N.; Messina, P.; Barth, J. V.; Kern, K. J. Am. Chem. Soc. 2003, 125, 10725.

(19) Stepanow, S.; Lingenfelder, M.; Dmitriev, A.; Spillmann, H.; Delvigne, E.; Lin, N.; Deng, X.; Cai, C.; Barth, J. V.; Kern, K. Nat. Mater. 2004, 3, 229.

(20) Lingenfelder, M.; Spillmann, H.; Dmitriev, A.; Stepanow, S.; Lin, N.; Barth, J. V.; Kern, K. Chem.-Eur. J. 2004, 10, 1913.
(21) Stepanow, S.; Lin, N.; Barth, J. V.; Kern, K. J. Phys. Chem. B 2006, 110, 23472.

(22) Stepanow, S.; Lin, N.; Barth, J. V.; Kern, K. Chem. Commun. 2006, 2153

(23) Stepanow, S.; Lin, N.; Barth, J. V. J. Phys.: Condens. Matter 2008, 20,184002 .

(24) Decker, R.; Schlickum, U.; Klappenberger, F.; Zoppellaro, G.; Klyatskaya, S.; Ruben, M.; Barth, J. V.; Brune, H. Appl. Phys. Lett. 2008, 93, 243102.

(25) Shi, Z.; Lin, N. J. Am. Chem. Soc. 2009, 131, 5376.

(26) Heim, D.; Seufert, K.; Auwärter, W.; Aurisicchio, C.; Fabbro, C.; Bonifazi, D.; Barth, J. V. Nano Lett. 2010, 10, 122.

(27) Schull, G.; Douillard, L.; Fiorini-Debuisschert, C.; Charra, F.; Mathevet, F.; Kreher, D.; Attias, A.-J. Nano Lett. 2006, 6, 1360.

(28) Spillmann, H.; Kiebele, A.; Jung, T. A.; Bonifazi, D.; Cheng, F.; Diederich, F. Adv. Mater. 2006, 18, 275.

(29) Mena-Osteritz, E.; Bäuerle, P. Adv. Mater. 2006, 18, 447.

(30) Tahara, K.; Furukawa, S.; Uji-i, H.; Uchino, T.; Ichikawa, T.; Zhang, J.; Mamdouh, W.; Sonoda, M.; De Schryver, F. C.; De Feyter, S.; Tobe, Y. J. Am. Chem. Soc. 2006, 128, 16613.

(31) Zwaneveld, N. A. A.; Pawlak, R.; Abel, M.; Catalin, D.; Gigmes, D.; Bertin, D.; Porte, L. J. Am. Chem. Soc. 2008, 130, 6678.

(32) Weigelt, S.; Bombis, C.; Busse, C.; Knudsen, M. M.; Gothelf, K. V.; Laegsgaard, E.; Besenbacher, F.; Linderoth, T. R. ACS Nano 2008, 2, 651.

(33) Schlickum, U.; Decker, R.; Klappenberger, F.; Zoppellaro, G.; Klyatskaya, S.; Auwärter, W.; Neppl, S.; Kern, K.; Brune, H.; Ruben, M.; Barth, J. V. J. Am. Chem. Soc. 2008, 130, 11778.

(34) Kühne, D.; Decker, R.; Klappenberger, F.; Schlickum, U.; Brune, H.; Zoppellaro, G.; Klyatskaya, S.; Ruben, M.; Barth, J. V. J. Phys. Chem. C 2009, 113, 17851

(35) Schlickum, U.; Decker, R.; Klappenberger, F.; Zoppellaro, G.; Klyatskaya, S.; Ruben, M.; Silanes, I.; Arnau, A.; Kern, K.; Brune, H.; Barth, J. V. Nano Lett. 2007, 7, 3813.

(36) Kühne, D.; Klappenberger, F.; Schlickum, U.; Decker, R.; Brune, H.; Klyatskaya, S.; Ruben, M.; Barth, J. V. J. Am. Chem. Soc. 2009, 131, 3881.

(37) Stepanow, S.; Lin, N.; Payer, D.; Schlickum, U.; Klappenberger, F.; Zoppellaro, G.; Ruben, M.; Brune, H.; Barth, J. V.; Kern, K. Angew. Chem., Int. Ed. 2007, 46, 710.

(38) Clair, S.; Pons, S.; Seitsonen, A. P.; Brune, H.; Kern, K.; Barth, J. V. J. Phys. Chem. B 2004, 108, 19392.

(39) Crommie, M. F.; Lutz, C. P.; Eigler, D. M. Nature 1993, 363, 524. (40) Messina, P.; Dmitriev, A.; Lin, N.; Spillmann, H.; Abel, M.; Barth, J. V.; Kern, K. J. Am. Chem. Soc. 2002, 124, 14000.

(41) Clair, S.; Pons, S.; Fabris, S.; Baroni, S.; Brune, H.; Kern, K.; Barth, J. V. J. Phys. Chem. B 2006, 110, 5627.

(42) Seitsonen, A. P.; Lingenfelder, M.; Spillmann, H.; Dmitriev, A.; Stepanow, S.; Lin, N.; Kern, K.; Barth, J. V. J. Am. Chem. Soc. 2006, 126, 5634.

(43) Tait, S. L.; Langner, A.; Lin, N.; Stepanow, S.; Chandrasekar, R.; Ruben, M.; Kern, K. J. Phys. Chem. C 2007, 111, 10982.

(44) Klappenberger, F.; Weber-Bargioni, A.; Auwärter, W.; Marschall, M.; Schiffrin, A.; Barth, J. V. J. Chem. Phys. 2008, 129, 214702.

(45) Marschall, M.; Reichert, J.; Weber-Bargioni, A.; Seufert, K.; Auwärter, W.; Klyatskaya, S.; Zoppellaro, G.; Ruben, M.; Barth, J. V. Nat. Chem. 2010, 2, 131.

JP104518H 


\title{
Supporting Information
}

\section{Surface-confined metal-organic nanostructures from Co- directed assembly of linear terphenyl-dicarbonitrile linkers on $\operatorname{Ag}(111)$}

\author{
U. Schlickum ${ }^{1 *}$, F. Klappenberger ${ }^{2}$, R. Decker ${ }^{1}$, G. Zoppellaro ${ }^{3}$, S. Klyatskaya ${ }^{3}$, \\ M. Ruben ${ }^{3^{*}}$, K. Kern ${ }^{1,4}$, H. Brune ${ }^{1}$, \& J.V. Barth ${ }^{2^{*}}$ \\ [1] Institute of Condensed Matter Physics, Ecole Polytechnique Fédérale de Lausanne, \\ $\mathrm{CH}-1015$ Lausanne, Switzerland \\ [2] Physik Department E20, Technische Universität München, D-85748 Garching, Germany

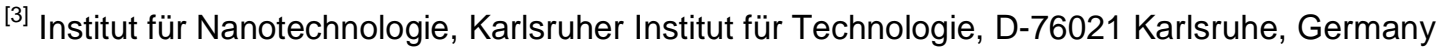 \\ ${ }^{[4]}$ Max-Planck-Institut für Festkörperforschung, D-70569 Stuttgart, Germany
}

The presence of two domains of the honeycomb nanomesh rotated by $30^{\circ}$ with respect to each other and their orientation with respect to the atomic $\operatorname{Ag}(111)$ lattice can also be seen by low energy electron diffraction (LEED) images shown in Figure S1. The spots of the atomic $\operatorname{Ag}(111)$ lattice are presented in Figure S1a and spots resulting from the honeycomb superlattice in Figure S1b. In the latter the metal-organic superstructure forms six bright spots and in between six weaker spots, indicating that one domain occupies more of the surface area. The two rotational domains are in accordance with the STM images. From STM images and the LEED pattern we conclude that the domain having the molecules aligned along $\langle 11-2\rangle$ is more frequently present. This preferred orientation is also observed for the para-hexaphenyl-dicarbonitrile-based honeycomb nanomeshes. 

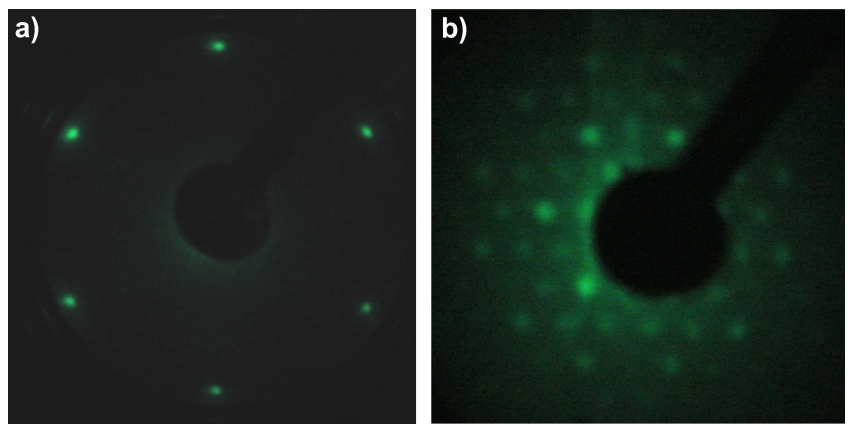

Figure S1a) LEED pattern showing principle spots of the $\mathrm{Ag}(111)$ surface at an energy of $53 \mathrm{eV}$. b) LEED pattern of the honeycomb superstructures formed by $\mathrm{NC}-\mathrm{Ph}_{3}-\mathrm{CN}$ and $\mathrm{Co}$ atoms taken at an energy of $8 \mathrm{eV}$.

Close to the coverage of one monolayer of the organic $\mathrm{NC}-\mathrm{Ph}_{3}-\mathrm{CN}$ molecules on the $\mathrm{Ag}(111)$ surface, a room temperature stable pure organic network is formed (see Figure S2). This network pattern is similar to the chevron pattern observed at low temperatures for submonolayer coverage. Since for small coverage the molecules are very mobile on the surface at room temeprature, we can conclude that the stability of a monolayer results from i) a hindered in plane diffusion due to space limitations and ii) a blocked vertical diffusion of the molecules prohibiting the formation of molecular multilayers.

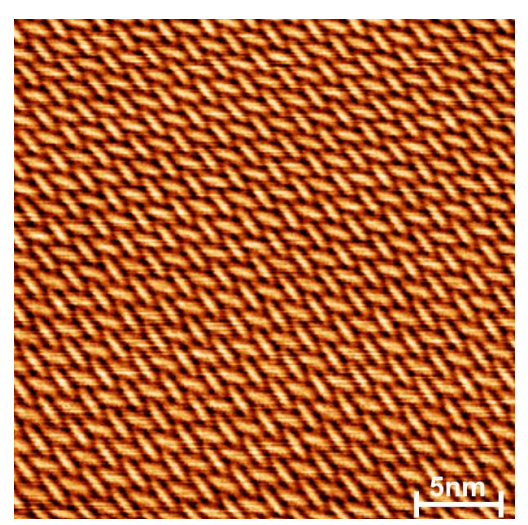

Figure S2: Pure organic phase that is stable at room temperature for coverages close to one monolayer. The STM image is taken at $300 \mathrm{~K}$.

Acknowledgement: We thank Willi Auwärter, Markus Etzkorn, Stefano Rusponi, Knud Seufert, Pardeep Thakur and the European Synchrotron Radiation Facility for their help with the LEED and the room temperature STM image. Work was supported by the European Science Foundation Collaborative Research Programme FunSMARTs. 